\title{
Biochemical correlates of neuropsychiatric illness in maple syrup urine disease
}

\author{
Emilie R. Muelly, ${ }^{1,2}$ Gregory J. Moore,1,2,3,4 Scott C. Bunce,1,2 Julie Mack, ${ }^{1,3}$ Don C. Bigler, ${ }^{1,2,5}$
} D. Holmes Morton, $6,7,8$ and Kevin A. Strauss $6,7,8$

\begin{abstract}
${ }_{1}^{1}$ Penn State Hershey Neurosciences Institute, 2Department of Psychiatry, and 3Department of Radiology, College of Medicine, Pennsylvania State University, Hershey, Pennsylvania, USA. ${ }^{4}$ Department of Radiology, Geisinger Health System, Danville, Pennsylvania, USA. ${ }^{5}$ AREVA NDE-Solutions, North America, AREVA Inc., Lynchburg, Virginia, USA. ${ }^{6}$ Clinic for Special Children, Strasburg, Pennsylvania, USA. 'Franklin and Marshall College,
\end{abstract}

Lancaster, Pennsylvania, USA. ${ }^{8}$ Lancaster General Hospital, Lancaster, Pennsylvania, USA.

\begin{abstract}
Maple syrup urine disease (MSUD) is an inherited disorder of branched chain amino acid metabolism presenting with neonatal encephalopathy, episodic metabolic decompensation, and chronic amino acid imbalances. Dietary management enables survival and reduces risk of acute crises. Liver transplantation has emerged as an effective way to eliminate acute decompensation risk. Psychiatric illness is a reported MSUD complication, but has not been well characterized and remains poorly understood. We report the prevalence and characteristics of neuropsychiatric problems among 37 classical MSUD patients (ages 5-35 years, 26 on dietary therapy, 11 after liver transplantation) and explore their underlying mechanisms. Compared with 26 age-matched controls, MSUD patients were at higher risk for disorders of cognition, attention, and mood. Using quantitative proton magnetic resonance spectroscopy, we found lower brain glutamate, $\mathrm{N}$-acetylaspartate (NAA), and creatine concentrations in MSUD patients, which correlated with specific neuropsychiatric outcomes. Asymptomatic neonatal course and stringent longitudinal biochemical control proved fundamental to optimizing long-term mental health. Neuropsychiatric morbidity and neurochemistry were similar among transplanted and nontransplanted MSUD patients. In conclusion, amino acid dysregulation results in aberrant neural networks with neurochemical deficiencies that persist after transplant and correlate with neuropsychiatric morbidities. These findings may provide insight into general mechanisms of psychiatric illness.
\end{abstract}

\section{Introduction}

Hereditary disorders that cause neuropsychiatric sequelae are a window into the biological foundations of mental illness. One such condition, branched chain ketoacid dehydrogenase (BCKDH) deficiency, also known as maple syrup urine disease (MSUD), leads to accumulation of branched chain amino acids (BCAAs) (leucine, isoleucine, and valine) and their derivative $\alpha$-ketoacids in blood and tissues. Patients with the most severe form, classical MSUD, may appear normal at birth, but develop acute metabolic decompensation within the first weeks of life. If not appropriately diagnosed and treated, rapid elevation of circulating leucine and its ketoacid, $\alpha$-ketoisocaproate (aKIC), cause encephalopathy and life-threatening brain swelling (1). After the neonatal period, careful dietary management limits episodic decompensations to a variable extent, improves amino acid nutrition, and greatly reduces morbidity and mortality (2). Liver transplantation has recently emerged as an effective means to eliminate metabolic volatility and the risk of cerebral edema (3).

As treatment for MSUD improves, survival improves in parallel, and physicians now manage a growing number of adolescents and adults with classical MSUD. Mental health outcomes and their relation to treatment variables have not been fully characterized in this population. However, anecdotal reports suggest that aging MSUD patients are at high risk for chronic neuropsychiatric problems such as attention deficit disorder, depression, and anxiety (1). Although liver transplantation has proven highly effective for preventing acute crises, available evi-

Conflict of interest: The authors have declared that no conflict of interest exists. Citation for this article: J Clin Invest. 2013;123(4):1809-1820. doi:10.1172/JCI67217. dence suggests it may not improve intelligence quotient (IQ) or reverse psychiatric disease $(4,5)$.

Chronic neuropsychiatric sequelae of MSUD are likely caused by several interacting mechanisms also thought to be responsible for acute neurotoxicity. Proposed mechanisms of neurotoxicity include unbalanced cerebral essential amino acid uptake, neurotransmitter deficiencies, energy deprivation, and osmotic dysregulation. In MSUD, branched chain ketoacid metabolism is blocked by a dysfunctional BCKDH, causing concentrations of upstream aKIC and leucine to increase. Hyperleucinemia inhibits the transport of tyrosine, tryptophan, and other essential amino acids across the blood-brain barrier and thereby limits substrate availability for cerebral catecholamine, serotonin, and protein synthesis. Accumulation of aKIC favors synthesis of leucine in the bidirectional transaminase reaction, consuming glutamate. Glutamate is an important metabolic currency that is used as a neurotransmitter as well as a source of energy. Transaminases in brain tissue normally convert leucine to $\alpha$-ketoglutarate to supply nitrogen to the cerebral glutamate pool $(6,7)$. Elevated aKIC - the ketoacid derivative of leucine - reverses the net direction of nitrogen flow and thus depletes the brain of glutamate $(6,7)$. Consistent with these mechanisms, reduced cerebral dopamine and glutamate levels have been observed in experimental MSUD animals $(8,9)$ and postmortem brain tissue from a child who died of leucine intoxication (10).

Supporting the energy inhibition hypothesis, BCKDHA knockout mouse brain has low pyruvate, ATP, and phosphocreatine, and high $\alpha$-ketoglutarate, lactate, and alanine (9). In human MSUD patients, magnetic resonance spectroscopy (MRS) studies demonstrate reversible lactate elevation and $\mathrm{N}$-acetylaspartate (NAA) depletion during 


\section{Table 1}

Neurochemical quantification in maple syrup urine disease

\begin{tabular}{|c|c|c|c|c|c|c|}
\hline & $\begin{array}{c}\text { Controls } \\
\text { mean (SD) }\end{array}$ & $\begin{array}{l}\text { MSUD diet } \\
\text { mean (SD) }\end{array}$ & $\begin{array}{l}\text { MSUD transplant } \\
\text { mean (SD) }\end{array}$ & $\boldsymbol{F}$ & $P$ value & $\begin{array}{l}\text { Post } \\
\text { hoc }^{B}\end{array}$ \\
\hline \multicolumn{7}{|l|}{ Left basal gangliac } \\
\hline Creatine & $8.24(1.23)$ & $8.34(1.16)$ & $8.10(0.70)$ & 0.25 & NS & \\
\hline NAA & $8.88(1.91)$ & $8.21(1.75)$ & $8.34(1.27)$ & 1.21 & NS & \\
\hline Choline compounds & 2.020 .37 & $2.25(0.33)$ & $2.15(0.27)$ & 2.29 & NS & \\
\hline Myo-inositol & $3.39(0.83)$ & $3.68(0.89)$ & $3.50(0.51)$ & 0.94 & NS & \\
\hline Glutamate & $8.07(1.42)$ & $6.37(1.16)$ & $6.42(1.06)$ & 13.22 & $<0.001$ & $a, b$ \\
\hline \multicolumn{7}{|c|}{ Prefrontal and anterior cingulate cortex ${ }^{\mathrm{D}}$} \\
\hline Creatine & $7.86(0.81)$ & $6.85(1.71)$ & $7.70(0.82)$ & 3.49 & 0.04 & a \\
\hline NAA & $9.56(1.57)$ & $8.11(2.22)$ & $8.47(1.55)$ & 4.91 & 0.01 & a \\
\hline Choline compounds & $1.91(0.27)$ & $1.98(0.58)$ & $1.97(0.43)$ & 0.16 & NS & \\
\hline Myo-inositol & $5.53(1.16)$ & $4.75(1.35)$ & $5.94(0.94)$ & 2.67 & 0.08 & \\
\hline Glutamate & $10.68(1.58)$ & $7.41(2.10)$ & $8.64(0.90)$ & 19.08 & $<0.001$ & $a, b$ \\
\hline \multicolumn{7}{|c|}{ Right parietal white matter ${ }^{\mathrm{E}}$} \\
\hline Creatine & $4.95(0.72)$ & $4.38(0.38)$ & $4.43(0.51)$ & 6.74 & 0.002 & $a, b$ \\
\hline NAA & $8.41(1.09)$ & $7.82(0.95)$ & $7.43(1.37)$ & 3.69 & 0.03 & $\mathrm{~b}$ \\
\hline Choline compounds & $1.70(0.24)$ & $1.74(0.29)$ & $1.65(0.31)$ & 0.02 & NS & \\
\hline Myo-inositol & $3.69(0.77)$ & $3.76(0.97)$ & $3.58(0.90)$ & 0.11 & NS & \\
\hline Glutamate & $5.96(1.30)$ & $4.65(0.68)$ & $5.05(0.67)$ & 9.65 & $<0.001$ & $a, b$ \\
\hline
\end{tabular}

AMean values presented in millimoles per kilogram of wet weight. BSignificant group differences in post hoc comparisons for (a) MSUD diet versus controls and (b) MSUD transplant versus controls. There were no significant differences between MSUD diet and MSUD transplant groups. CDegrees of freedom =

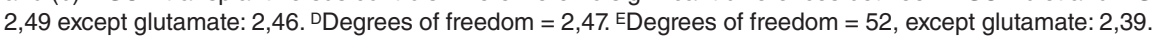

acute encephalopathic crises, reflecting transient compromise of cerebral mitochondrial function (11-13). Cerebral edema during acute crises consists of both a global vasogenic edema thought to be related to blood-brain barrier compromise and "MSUD edema," which is thought to be intramyelinic, cytotoxic edema secondary to energetic and osmotic dysregulation $(12,14)$. Qualitatively similar but less marked mechanisms may also contribute to chronic symptoms in patients considered metabolically stable.

These factors prompted us to characterize neuropsychiatric outcomes in MSUD patients and to determine how they correlate with clinical course, management variables, indices of cerebral essential amino acid nutrition, treatment strategy, and neurochemistry.

\section{Results}

Neurochemistry. MSUD patients had low glutamate in all brain regions (Table 1). Glutamate correlated inversely with plasma leucine levels (basal ganglia: $r_{s}=-0.37$, where $s$ indicates Spearman, degrees of freedom $[\mathrm{DF}]=41, P=0.009$; cortex: $r_{s}=-0.48, \mathrm{DF}=43$; $P<0.001$ ) and calculated cerebral leucine influx (basal ganglia: $r_{s}=-0.39, \mathrm{DF}=36 ; P=0.01 ;$ cortex: $r_{s}=-0.43, \mathrm{DF}=39 ; P=0.003$; Figure 1). Patients also had deficits of NAA and creatine in the anterior cingulate cortex and parietal white matter. No medication class was significant when included as a covariate $(P>0.05)$. Neurochemistry was unaffected by liver transplantation (Table 1$)$. Total brain volumes did not differ between groups $\left(F_{2,58}=0.30\right.$, where $F=F$ distribution; $P=0.75)$, nor did they correlate with any neuropsychiatric outcome measures $(P>0.05)$. Total brain volume correlated with concentrations of parietal white matter choline compounds $\left(r_{s}=-0.30, \mathrm{DF}=52 ; P=0.02\right)$ and creatine $\left(r_{s}=-0.36\right.$, $\mathrm{DF}=52 ; P=0.006)$, but no other neurometabolites.

Mood and anxiety. Relative to controls, MSUD patients had more depression, anxiety, inattention, and impulsivity. Together, these conditions reached a cumulative lifetime incidence of $83 \%$ by age
36 years (Figure $2 \mathrm{~A}$ and Table 2). When compared with MSUD patients who remained clinically asymptomatic throughout the newborn period, neonates who were encephalopathic at the time of diagnosis were 5 and 10 times more likely, respectively, to later suffer from anxiety and depression (Figure 2B and Table 3). Measures of depression and anxiety did not correlate strongly with indices of lifetime metabolic control (Table 4), but mood disturbances correlated with cortical myo-inositol, NAA, and glutamate (Table 5).

Intelligence and global function. Compared with controls (full scale IQ $106 \pm 15)$, full scale IQ was lower in MSUD patients treated with diet $(81 \pm 19)$ or transplant $(90 \pm 15)$, but these groups did not differ from one another (Table 2). Global assessment of function scores was also lower in both MSUD groups. Among all MSUD subjects, there was no correlation between full scale IQ and age $\left(r_{s}=-0.26, P=0.14\right)$. However, there was an inverse correlation between age and IQ within the subgroup of Mennonite patients (Figure 3A and Table 3). Neonatal encephalopathy did not affect risk for mental retardation, but was associated with a 4-fold higher risk of global functional impairment $(P=0.05$; Table 3$)$. Measures of intelligence correlated inversely to average lifetime plasma leucine and its concentration ratio to valine (an indirect index of cerebral valine uptake; Figure 3B) and positively correlated with the frequency of amino acid monitoring (Table 4). Prefrontal cortical myo-inositol and choline showed the strongest correlations with intelligence. An interaction among group, basal ganglia NAA concentration, and performance IQ indicated that the association between metabolite concentration and outcome was more positive for MSUD patients on diet relative to the other groups $\left(F_{2,43}=5.17, P=0.01\right.$; Control $\beta$ weight $(\beta)=-0.22$, MSUD-diet $\beta=0.34$, MSUD-transplant $\beta=-0.23$; Figure $3 C$ ).

Attention and hyperactivity. Cumulative lifetime incidence of attention deficit and hyperactivity disorder (ADHD) was 54\% among MSUD patients on dietary therapy, $82 \%$ among those treated with 


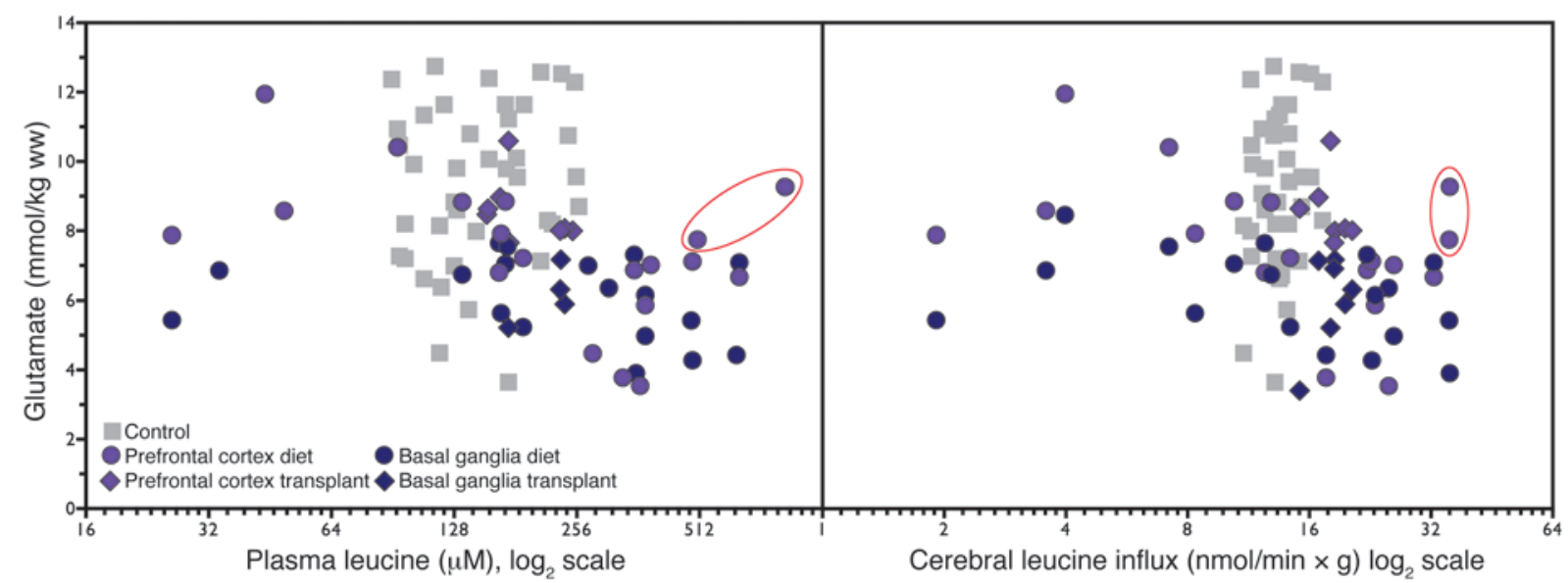

Figure 1

Glutamate (in mmol/kg wet weight, ww) in the prefrontal and anterior cingulate cortices (violet) and basal ganglia (blue) region inversely correlated with ambient plasma leucine (left panel) and the calculated cerebral leucine influx (right panel) in MSUD patients on diet (circles) or after liver transplantation (diamonds). Control subjects are represented as gray squares. The 2 MSUD diet patients who demonstrate relatively higher cortical glutamate levels despite having the highest estimated leucine influx are brothers (circled in red).

transplant, and unrelated to condition at diagnosis. Younger participants had higher ADHD symptom ratings, as might be expected given the natural course of ADHD (Figure 4A and Table 4). Surprisingly, higher ADHD scores were associated with lower average lifetime plasma leucine and higher lifetime tyrosine (Figure 4B and Table 4). However, post hoc analysis suggested a dichotomy in the leucine-attention relationship based on ADHD diagnosis. Specifically, MSUD patients with ADHD had a stronger positive correlation between plasma leucine levels and symptom severity compared with those without an ADHD diagnosis (ADHD lifetime diagnosis $\times$ leucine interaction, $F_{1,23}=5.37, P=0.02$; Figure $4 C)$. Several neurochemical concentrations correlated with ADHD symptom severity, including midline prefrontal and anterior cingulate cortex NAA (Figure 4D and Table 5).

\section{Discussion}

Over the last 2 decades, deeper understanding and better control of MSUD neurotoxicity has had a decisive impact on patient out- comes $(2,4,15-17)$. Reviewing clinical outcomes of the 20 Northeastern Mennonite children born with classical MSUD between 1963 and 1984, we found that 11 (55\%) died of cerebral edema by age 9 and all survivors became intellectually disabled. Since comprehensive MSUD services were established in Lancaster County in 1989, outcomes have markedly improved and there have been no deaths. We attribute this to advances in diagnosis, treatment, and medical food design $(2,15,16)$. With longer survival, our focus has shifted to the more subtle, chronic neurological sequelae of MSUD.

Evidence suggests that, over the life span of an MSUD patient, at least 3 mechanisms threaten brain structure and function: (a) cerebral essential amino acid deficiencies due to unbalanced blood-to-brain transport $(2,9,18,19)$; (b) depletion of neurotransmitters $(2,8-10,20-22)$; and (c) inhibition of mitochondrial enzymes and the respiratory chain (Figure 5 and refs. 9, 23-26). Although these mechanisms have been explored in acute settings, they may also contribute to chronic sequelae. Specific to neuropsychiatric illness, hyperleucinemia may adversely affect
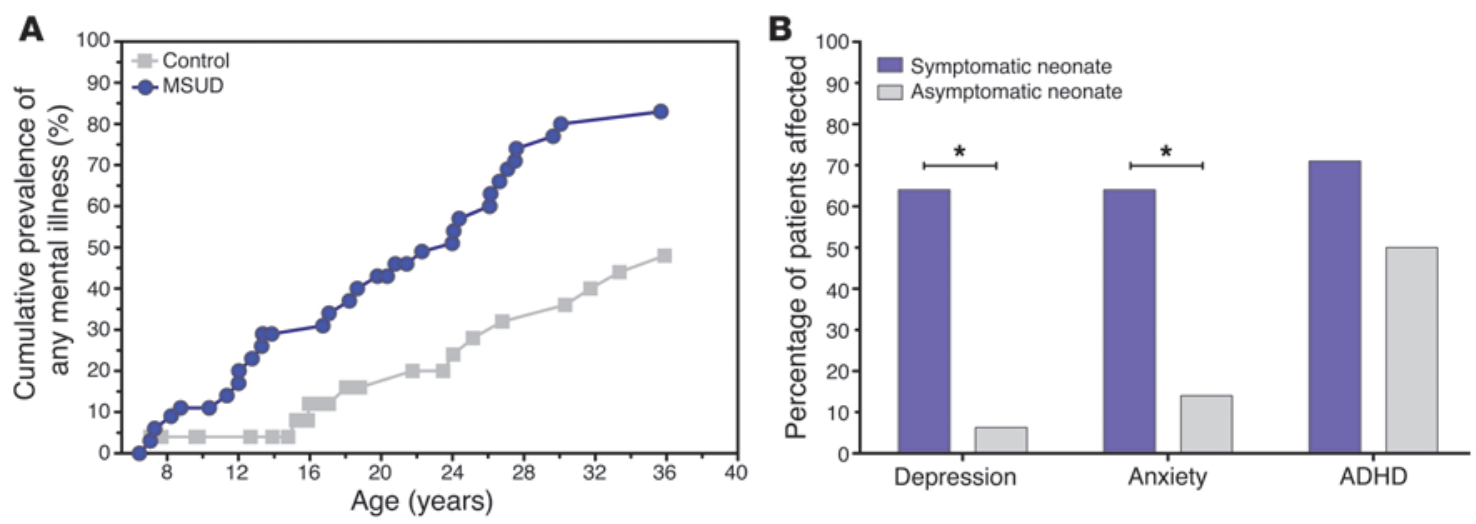

Figure 2

Cumulative prevalence and neonatal risks associated with neuropsychiatric outcome in classical MSUD patients. The cumulative lifetime incidence of mental illness (depression, anxiety disorders, ADHD) among all MSUD patients reached $83 \%$ by age 36 (A). Neonatal encephalopathy was a strong predictor of mood disorders, but not mental retardation or ADHD, later in life $(B) .{ }^{*} P<0.05$. 
Table 2

Population characteristics and neuropsychiatry in MSUD

\begin{tabular}{|c|c|c|c|c|c|c|}
\hline & $\begin{array}{l}\text { Controls } \\
(n=26)\end{array}$ & $\begin{array}{l}\text { MSUD diet } \\
\quad(n=24)\end{array}$ & $\begin{array}{l}\text { MSUD transplant } \\
\qquad(n=11)\end{array}$ & $F^{A}$ & $P$ value & $\begin{array}{l}\text { Post } \\
\text { hoc }^{B}\end{array}$ \\
\hline \multicolumn{7}{|l|}{ Categorical measures, $n$ (\%) } \\
\hline Female & $12(46 \%)$ & $12(50 \%)$ & $4(36 \%)$ & & NS & \\
\hline \multicolumn{7}{|l|}{ Full scale IQ } \\
\hline$I Q<70($ mild MR) & $0(0 \%)$ & $7(29 \%)$ & $2(18 \%)$ & & 0.009 & a \\
\hline$I Q<80$ (borderline) & $0(0 \%)$ & $11(46 \%)$ & $3(27 \%)$ & & $<0.001$ & a \\
\hline \multicolumn{7}{|l|}{ Attention deficit hyperactivity disorder } \\
\hline Current & $5(19 \%)$ & $12(50 \%)$ & $6(55 \%)$ & & 0.04 & a \\
\hline Lifetime & $7(27 \%)$ & $13(54 \%)$ & $9(82 \%)$ & & 0.006 & $\mathrm{~b}$ \\
\hline \multicolumn{7}{|l|}{ Depression } \\
\hline Current & $1(4 \%)$ & $7(29 \%)$ & $2(18 \%)$ & & 0.04 & a \\
\hline Lifetime & $5(19 \%)$ & $10(42 \%)$ & $4(36 \%)$ & & NS & \\
\hline \multicolumn{7}{|l|}{ Anxiety } \\
\hline Current & $4(15 \%)$ & $10(42 \%)$ & $5(45 \%)$ & & NS & \\
\hline Lifetime & $8(31 \%)$ & $14(58 \%)$ & $6(55 \%)$ & & NS & \\
\hline \multicolumn{7}{|l|}{ Continuous measures, mean (SD) } \\
\hline Age $18.7(8)$ & $20.4(7.6)$ & $15.0(6.9)$ & 0.88 & NS & & \\
\hline Full scale $I Q$ & $106(15)$ & $81(19)$ & $90(15)$ & 12.76 & $<0.001$ & $a, b$ \\
\hline Performance IQ & $106(13)$ & $82(16)$ & $86(15)$ & 17.29 & $<0.001$ & $a, b$ \\
\hline Verbal IQ & $104(16)$ & $82(22)$ & $93(15)$ & 8.43 & 0.001 & $\mathrm{a}$ \\
\hline \multicolumn{7}{|l|}{ Depression scales } \\
\hline Beck Depression Inventory (adults) & $2.5(3.1)$ & $6.3(9)$ & $8.5(11.1)$ & 1.47 & NS & \\
\hline Beck Youth Inventory (T-score) & $46(7)$ & $43(6)$ & $44(8)$ & 0.50 & NS & \\
\hline Combined (z-score, children + adults) & $-0.55(0.82)$ & $-0.34(1.23)$ & $-0.29(1.3)$ & 0.40 & NS & \\
\hline \multicolumn{7}{|l|}{ Anxiety scales } \\
\hline Beck Depression Inventory (adults) & $5.0(5.4)$ & $7.7(8)$ & $13.8(16.3)$ & 1.67 & NS & \\
\hline Beck Youth Inventory (T-score) & $44(7)$ & $42(7)$ & $47(9)$ & 0.91 & NS & \\
\hline Combined (z-score, children + adults) & $-0.34(0.86)$ & $-0.22(0.96)$ & $-0.06(1.5)$ & 0.62 & NS & \\
\hline \multicolumn{7}{|c|}{ Attention deficits - Conner's Parent Rating DSM-IV Sub-scores (T-scores) } \\
\hline Total & $46(5)$ & $57(13)$ & $56(11)$ & 8.13 & 0.001 & $a, b$ \\
\hline Inattentive & $44(5)$ & $57(13)$ & $53(10)$ & 9.85 & $<0.01$ & a \\
\hline Hyperactive + impulsive & $46(5)$ & $58(14)$ & $57(11)$ & 7.98 & 0.001 & $a, b$ \\
\hline Global assessment of function & $85(8)$ & $72(12)$ & $74(13)$ & 8.07 & 0.001 & $a, b$ \\
\hline
\end{tabular}

${ }^{A}$ Degrees of freedom $=2,27$ for depression and anxiety symptoms; ranged 2,52 to 2,56 for IQ and Conner's testing. BSignificant group differences in post hoc comparisons for (a) MSUD diet versus controls and (b) MSUD transplant versus controls. There were no significant differences between MSUD diet and MSUD transplant groups.

mood and cognition by competitively inhibiting uptake of tyrosine and tryptophan by the nervous system, thereby limiting cerebral production of dopamine, norepinephrine, and serotonin. Glutamate is the most abundant neurotransmitter in the brain and mediates "fast" excitatory transmission at most central synapses. Acute and chronic depletions of glutamate observed in MSUD patients are expected to have substantial consequences for cognition and behavior. Neurotransmitter deficiencies can be a primary cause of psychiatric illness or entrain compensatory changes in receptor expression and synaptic structure that affect brain function. Neuronal energy depletion, osmotic dysregulation, and dysmyelination also likely contribute. These influences may take place on an ongoing basis or stem from developmental or cumulative aberrations of neurochemistry that lead to persistent modifications of receptor regulation and neurochemistry.

Neurochemistry. We show that the neurochemical deficiencies previously demonstrated in acutely ill MSUD patients and experimental animals may persist, although to a lesser degree, in the chronic state (Table 1) and cumulatively contribute to neuro- psychiatric morbidity (Table 3). Evidence suggests that increased intracranial aKIC can reverse the overall transamination flow of leucine nitrogen to glutamate, thus depleting cerebral glutamate pools $(6,7,20,21,27)$. This mechanism appears to operate even within day-to-day fluctuations of aKIC found in MSUD patients considered metabolically stable. aKIC may have additional toxic

\section{Table 3}

Lifetime relative risks based on condition at the time of diagnosis

\begin{tabular}{lcc} 
III versus well & $\begin{array}{c}\text { Relative } \\
\text { risk }\end{array}$ & $\begin{array}{c}\text { Fisher's Exact } \\
\boldsymbol{P} \text { value }\end{array}$ \\
at diagnosis & 10.3 & 0.001 \\
Depression & 5.1 & 0.007 \\
Anxiety & 4.0 & 0.05 \\
Global assessment of functioning $<70$ & 2.9 & 0.20 \\
Full scale IQ $<70$ & 1.4 & 0.28 \\
Attention deficit hyperactivity & & \\
\hline
\end{tabular}




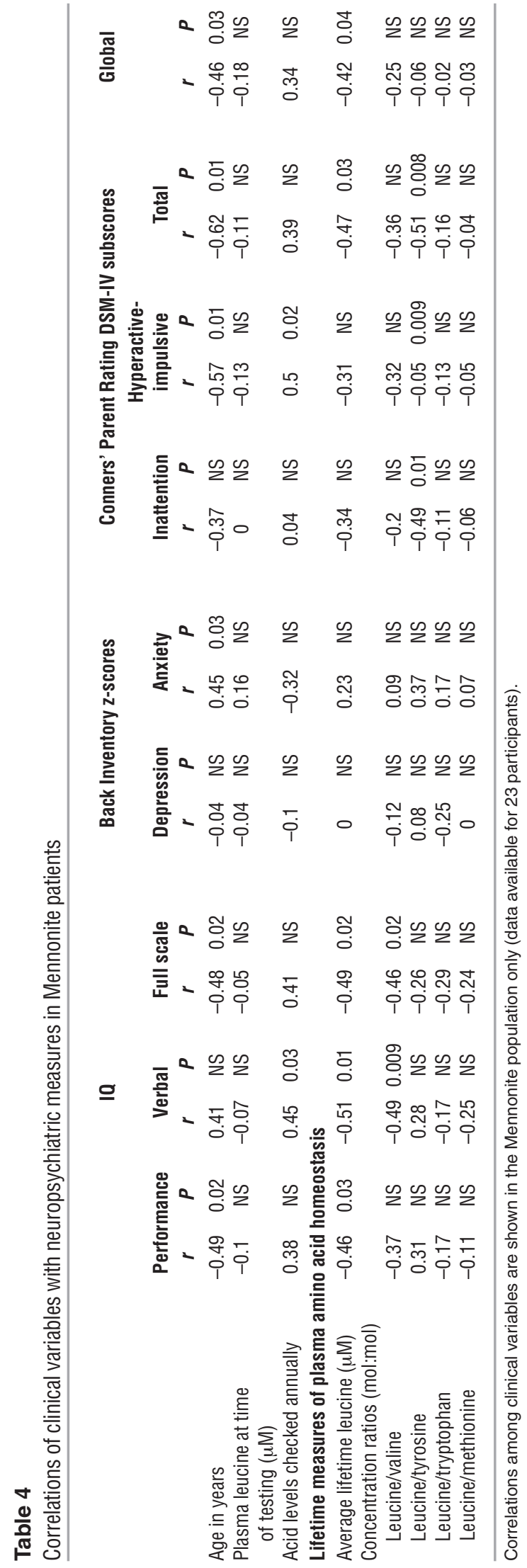

effects on brain metabolism that explain the reduced cerebral concentrations of NAA and creatine we observed in MSUD patients. In vitro, modestly elevated aKIC concentrations $(0.5 \mathrm{mM})$ uncouple mitochondria (26), and higher concentrations ( $2 \mathrm{mM}$ ) inhibit $\alpha$-ketoglutarate dehydrogenase and pyruvate dehydrogenase (25). Similar actions might interfere with mitochondrial NAA synthesis in vivo (Figure 5), particularly at higher ketoacid levels observed during acute crises. Cerebral ketoacid concentrations in living MSUD patients are unknown, but based on observed plasma concentrations and the known properties of the blood-brain monocarboxylate transporter, aKIC may frequently reach cerebral concentrations exceeding $0.5 \mathrm{mM}$ in vivo.

Alternatively, cortical NAA and creatine deficiency may simply reflect reduced neuronal density. Consistent with this hypothesis, one postmortem study demonstrated abnormal cytoarchitecture, thin dendrites, and decreased synaptic spine density in MSUD brain (28). Structural damage that decreases total tissue NAA and creatine content might accrue over many years, with greatest susceptibility during the early phase of brain development. Although total brain volumes did not differ across groups, we observed significant brain atrophy in some older MSUD patients (Figure 6), as has been reported in other neuroimaging studies (29). Our finding of reduced creatine suggests that creatine constancy should not be assumed for use as an internal reference in disease states.

In certain clinical settings, the relationship between neurochemistry and mental illness might be relatively specific. Other inborn errors (e.g., phenylketonuria, ornithine transcarbamoylase deficiency) entrain neurochemical states that may predispose to mental illness, but unusual physiologic properties of the bloodbrain barrier may protect a small subset of individuals and attenuate their risk for cognitive disability (30-32). A similar principle could apply to MSUD. In our study, 2 mentally healthy brothers with MSUD had elevated plasma leucine levels without proportionately decreased cortical glutamate (Figure 1). This may be traceable to Michaelis-Menten properties of the cerebral LAT1 transporter that influence intellectual outcome, as has been in proposed in phenylketonuria $(30,31)$.

Cognition, mood, and global function. The inverse relationship between IQ and age among Mennonite patients likely reflects cumulative improvements in medical care over the last 2 decades. Specifically, older patients may have lower IQs because they could not benefit early in life from the improved treatment regimens available today. These improvements include more accessible local outpatient and inpatient services, frequent home amino acid monitoring, outpatient "sick-day" management protocols, reduced hospitalization rates, and more rational formula design (Figure 7). However, other possible causes for potential fluctuations or trends in IQ over time should be investigated further through longitudinal studies beyond the scope of this report.

Despite important clinical progress, MSUD patients still have a high risk of neuropsychiatric disease that is not abrogated by liver transplant. Our results suggest that an asymptomatic newborn period, stringent leucine homeostasis, and cerebral essential amino acid (particularly valine) sufficiency are vital to long-term mental health and global function. Not directly addressed in our study, the experience of having a chronic illness may also contribute to depressed or anxious states. The relationship of mood instability to early brain growth is particularly striking; patients diagnosed as encephalopathic newborns were 5 and 10 times more likely to later develop anxiety and depression, respectively, than 


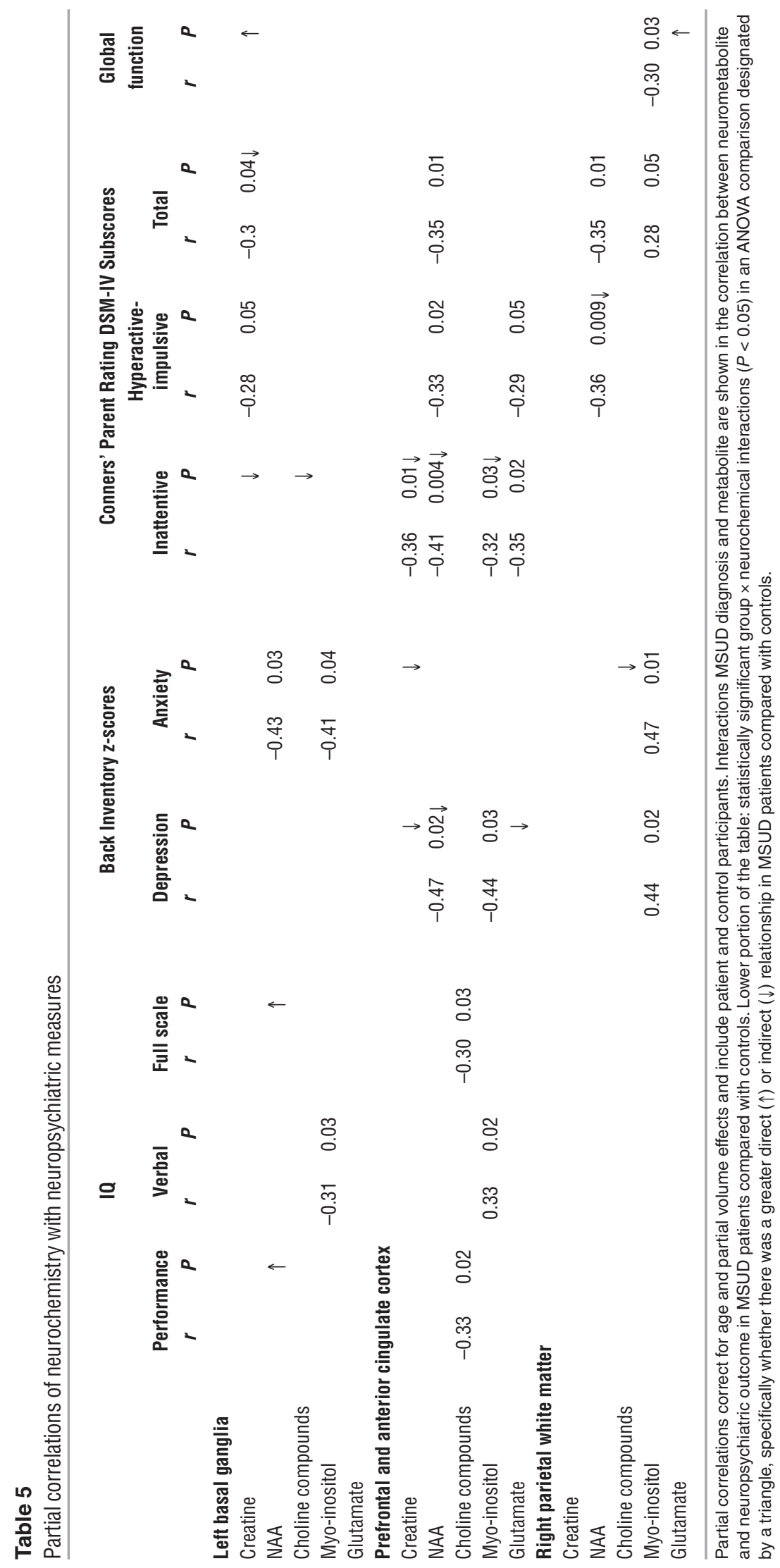



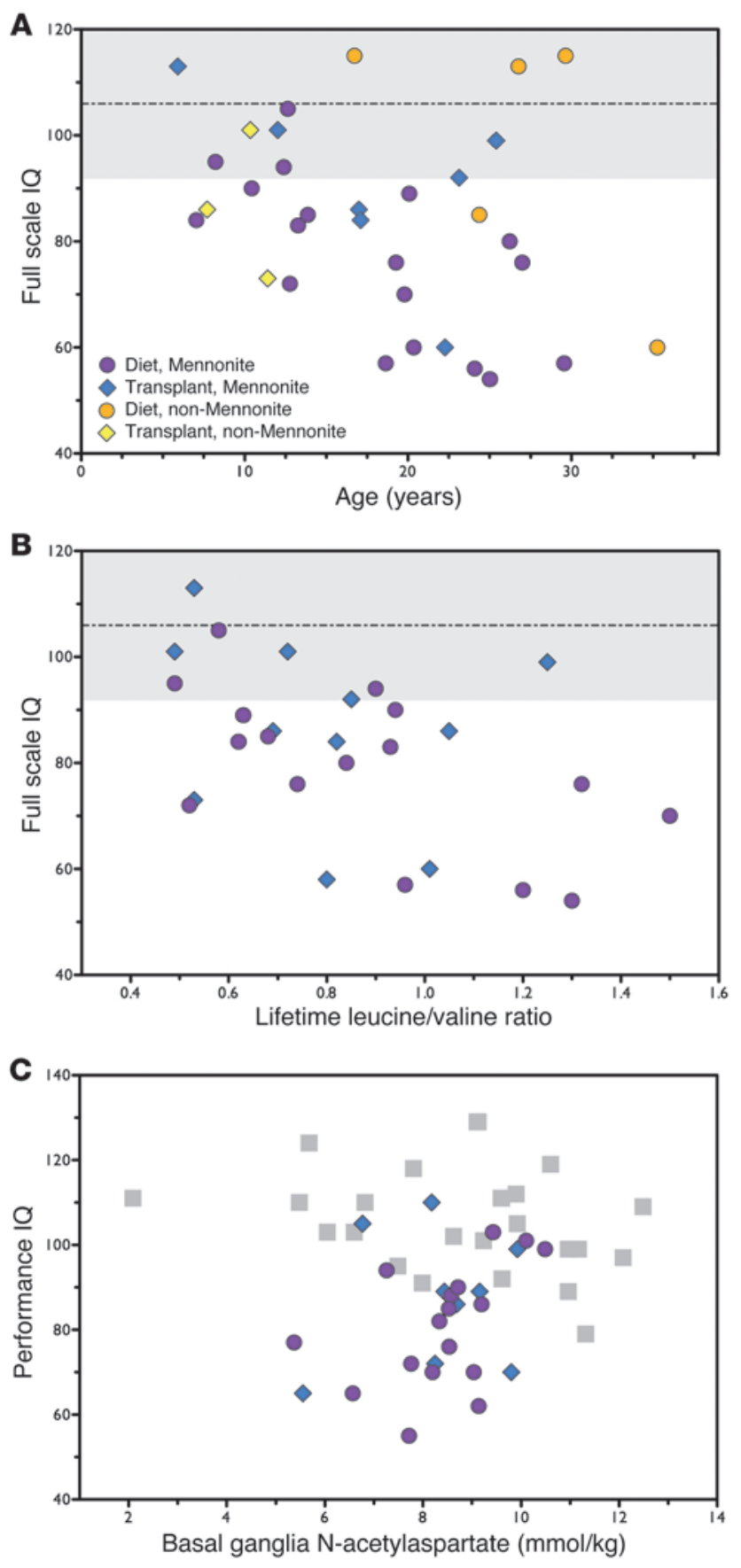

those who remained metabolically stable throughout the neonatal transition (Table 3). This suggests that early insults to the brain may have long-term consequences and highlights the importance of carrier testing and screening programs that allow for early identification and intervention.

In addition to cumulative cytostructural changes, ongoing neurochemistry may influence cognition and affect. Cerebral myo-inositol, important in cell growth and osmoregulation (33), correlates with both intelligence and mood. We did not detect differences of this metabolite among groups, but recurrent bouts of cerebral edema that accompany hyperleucinemia could drive dynamic changes of myo-inositol that influence higher cortical

\section{Figure 3}

Clinical and neurochemical correlates of IQ in classical MSUD patients. There was an inverse correlation between IQ and age among the subgroup of Mennonite patients (A). An inverse correlation between full scale IQ and lifetime plasma leucine/valine ratio was found (B). Cerebral metabolites, such as basal ganglia $\mathrm{N}$-acetylasparate, also correlated with various IQ measures (C). Symbols: Mennonite MSUD diet (violet circles); Mennonite MSUD transplant (blue diamonds); non-Mennonite MSUD diet (orange circles); non-Mennonite transplant (yellow diamonds); control subjects, (gray shaded areas [mean $\pm \mathrm{SD}$ ] or gray squares).

function. The association of affective disorders with prefrontal and anterior cingulate neurochemical deficiencies supports the notion that these regions play a role in regulating emotions (34). Interestingly, some relationships between neurochemistry and neuropsychiatric outcomes were stronger in MSUD patients compared with controls. Although MSUD patients did not have lower NAA levels in the basal ganglia than controls, a more positive relationship between this metabolite concentration and performance IQ existed for MSUD patients on diet compared with controls (Figure 3C). This could imply a greater reliance on neuronal energy for function in MSUD patients.

Attention and byperactivity. MSUD patients have an alarming lifetime risk of ADHD. Unlike intelligence and mood, ADHD subscores are highest in younger patients and are not strongly influenced by condition at diagnosis. Sibling controls also have a high prevalence of ADHD relative to the reported value of $9.6 \%-10.9 \%$ in the general population of Pennsylvania (35). This could reflect additional familial factors, but may also result from type II error.

ADHD in MSUD shows a complicated relationship to indices of lifetime metabolic control and time-of-testing neurochemistry. Patients with lower lifetime and recent leucine levels have higher ADHD scores. Surprisingly, high lifetime tyrosine and low plasma ratio of leucine to tyrosine - an indirect index of leucine inhibition on cerebral tyrosine influx (19) - are associated with ADHD. Tyrosine is the substrate for brain catecholamines, and although attention mechanisms have been linked to catecholaminergic signaling, the risk of inattention from higher cerebral tyrosine influx runs counter to current hypotheses $(19,36)$. This may relate to receptor modulation. Further exploration is warranted to better understand these relationships. Interestingly, our post hoc analysis shows that a subgroup of MSUD patients is more vulnerable to acute ADHD symptoms at times of moderate leucinemia (Figure 4D), reflecting genetic or developmental variations in susceptibility to plasma leucine dysregulation.

ADHD symptoms are also associated with low gray matter metabolites, particularly NAA and glutamate. The anterior cingulate cortex is active in situations that demand attention; however, little is known about anterior cingulate or midline prefrontal cortex neurochemistry in ADHD patients. One study did demonstrate an inverse association of ADHD with creatine and another with qualitative levels of glutamate and glutamine in these brain regions $(37,38)$. Additional studies of midline cortical neurochemistry could contribute more broadly to our understanding of mechanisms of attention.

As with mood, neurochemical correlates of inattention are more varied when comparing groups and include strong inverse correlations to cortical myo-inositol, creatine, and choline. The relative interaction between group and basal ganglia choline in 

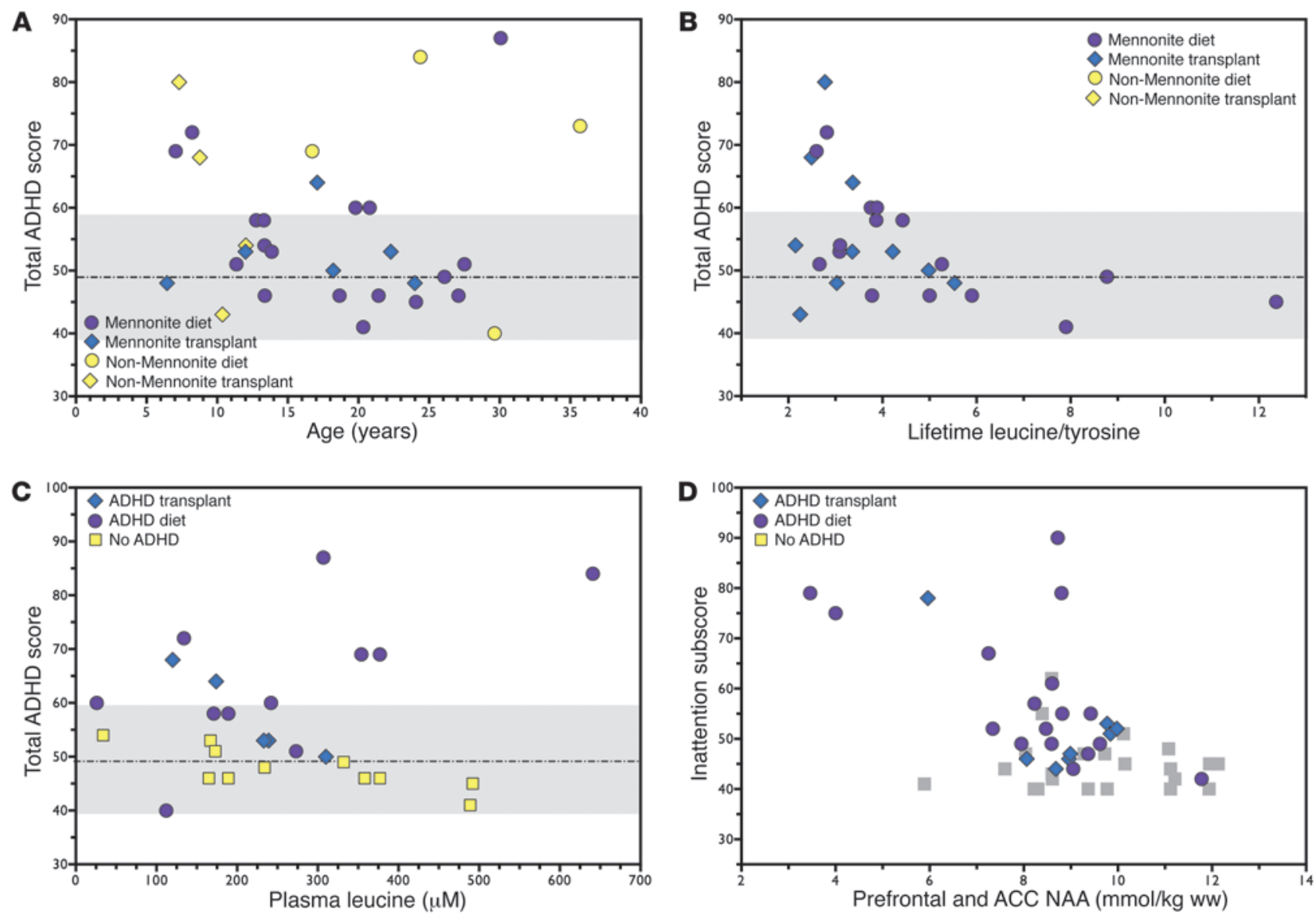

Figure 4

Clinical and neurochemical correlates of ADHD symptoms in MSUD patients. ADHD was inversely correlated to age (A) and directly related to the lifetime plasma leucine/tyrosine ratio (B). Plasma leucine at the time of the study does not correlate with ADHD symptoms scores. However, an interaction exists between plasma leucine and history of an ADHD diagnosis in predicting total ADHD ratings at the time of the study, such that in those patients who have ever had a diagnosis of ADHD, higher leucine levels corresponded with higher symptom ratings (C). Severity of inattention symptoms correlated with several metabolites, such as prefrontal and anterior cingulate (ACC) cortical NAA (D). Symbols: Mennonite MSUD diet (violet circles); Mennonite MSUD transplant (blue diamonds); non-Mennonite MSUD diet (yellow circles); non-Mennonite transplant (yellow diamonds); no ADHD (yellow squares); control subjects (gray shaded areas [mean $\pm \mathrm{SD}$ ] or gray squares).

predicting scores of inattention suggests that for MSUD patients, but not controls, higher choline compound levels are associated with improved attention. Higher choline compound signals could represent increased membrane turnover or acetylcholine pools (33). Together, these findings suggest that in MSUD, attention is a more dynamic phenomenon than intelligence and mood and could be affected by a broader network of neurochemical systems.

Liver transplantation. The persistence of psychiatric morbidity and neurochemical dysregulation following transplantation is unsettling but consistent with prior observations $(4,5)$. This suggests that neurostructural changes, acquired during cerebral development or longitudinally, may be relatively irreversible (28, 29). Moreover, persistent deficiency of cerebral BCKDH in transplant patients might continue to interfere with the neurochemical microenvironment despite normalization of systemic BCAA homeostasis. The current study, however, may have failed to detect meaningful differences due to the cross-sectional design, limited sample size, insensitivity of chosen tests, variable age, time since transplantation, or interindividual response differences.
Whatever the explanation, our results show clearly that liver transplant cannot be expected to eradicate all psychiatric sequelae of MSUD and does not eliminate the need for timely newborn screening and rigorous medical follow-up $(1,2,15)$. To the extent that liver transplant can stabilize BCAA homeostasis during the early phase of brain development, transplantation early in life will likely afford the most substantial long-term benefit (3).

Conclusion. The current study provides what we believe is the first comprehensive analysis of psychiatric comorbidity in MSUD and correlates these findings with immediate and lifetime biochemical and neurochemical variation. Our findings highlight the importance of early disease detection and metabolic control and the limitations of liver transplantation with respect to mental health outcomes. Specificity of correlations between metabolic control and neuropsychiatric morbidity underscores the value of using comprehensive models of cerebral essential amino acid nutrition, viewed over the arc of lifetime, to guide treatment. Fluctuations of systemic biochemistry within a reasonable framework of metabolic control show limited discernible relationship to neuropsy- 


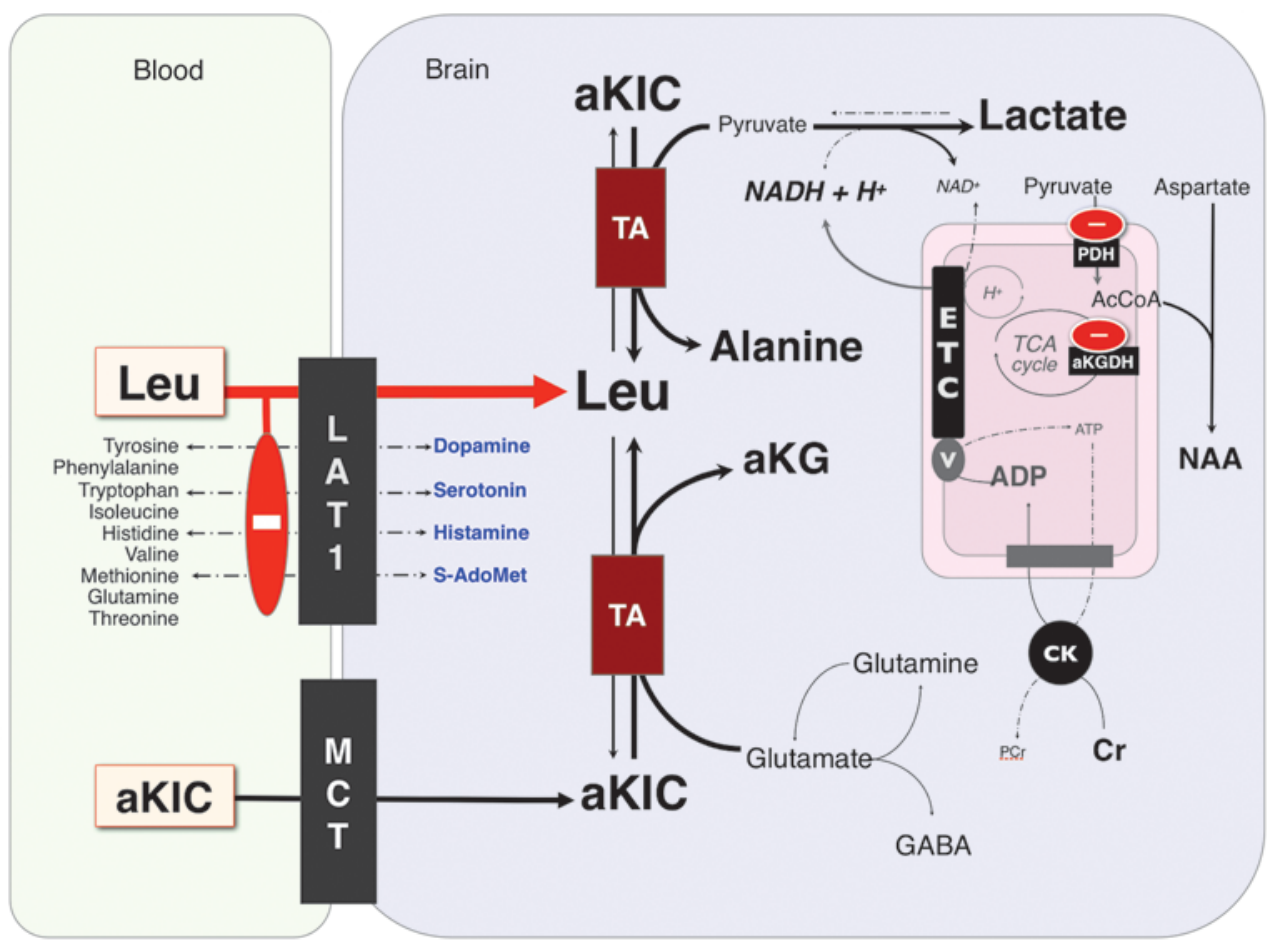

\section{Figure 5}

Theories of neurotoxic mechanisms of MSUD. At the blood-brain barrier, leucine, which has a low $K_{m}$ for LAT1, saturates the transporter and blocks uptake of its competitors tyrosine, phenylalanine, tryptophan, isoleucine, histamine, valine, methionine, glutamine, and threonine. Among these are precursors for neurotransmitters (dopamine, norepinephrine, serotonin, and histamine) and S-adenosylmethionine (S-AdoMet), the brain's major methyl donor. aKIC enters the brain via the monocarboxylate transporter (MCT) and reverses flux through cerebral transaminases (TA). This depletes brain glutamate, GABA, and glutamine while increasing production of leucine and $\alpha$-ketoglutarate (aKG). Glutamate and GABA are the most abundant excitatory and inhibitory neurotransmitters, respectively, in the human brain. MSUD encephalopathy may also block oxidative phosphorylation through an as yet unknown mechanism; in vitro data has implicated aKIC-mediated inhibition of pyruvate dehydrogenase $(\mathrm{PDH}), \alpha$-ketoglutarate dehydrogenase (aKGDH), and components of the electron transport chain (ETC). Impaired mitochondrial function can interfere with the production of NAA, which therefore serves as a marker for neuronal energy production. Additionally, energy from ATP is sometimes transferred to the creaine-phosphocreatine (Cr-PCr) system for later use.

chiatric outcome, whereas remote clinical history and long-term metabolic management have a substantial impact. Chronic neurochemical deficiencies and correlations of metabolic control and neurochemistry with neuropsychiatric outcomes provide insight into basic physiological mechanisms that may underlie chronic MSUD brain disease. Future work should address potential ways of preventing psychiatric comorbidity in MSUD and explore the relevance of the current findings to general psychiatry.

\section{Figure 6}

Cerebral atrophy in MSUD. A series of T2-weighted axial images from an MSUD participant who experienced poor metabolic control early in life (upper panel) and an age-matched control (lower panel) show the cerebral atrophy observed in some older patients who experienced prolonged amino acid imbalances during infancy or long periods of poor longitudinal metabolic control. There is a general loss of brain tissue at every level of the neturaxis, visible as thinner cortical gyri and cerebellar fossa, prominent sulci, and expansion of the perivascular spaces, particularly evident near the cortical surface and temporal lobes.

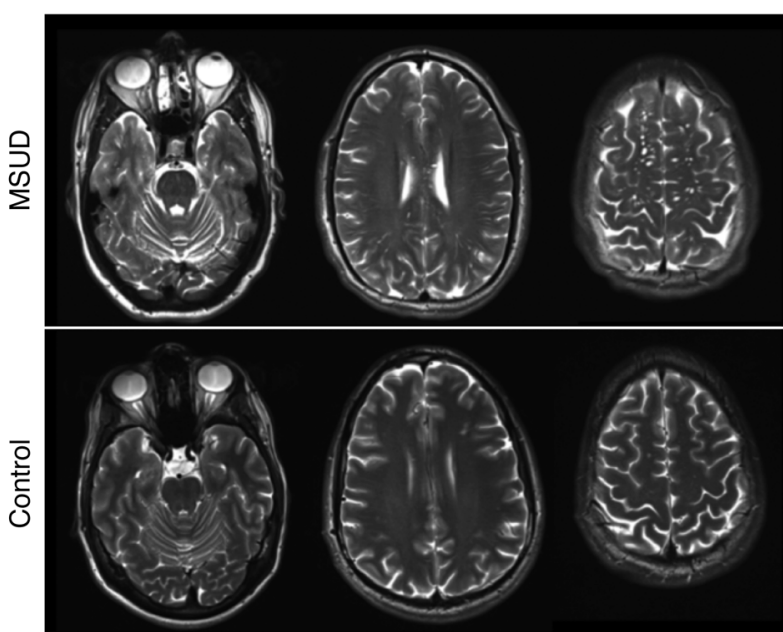




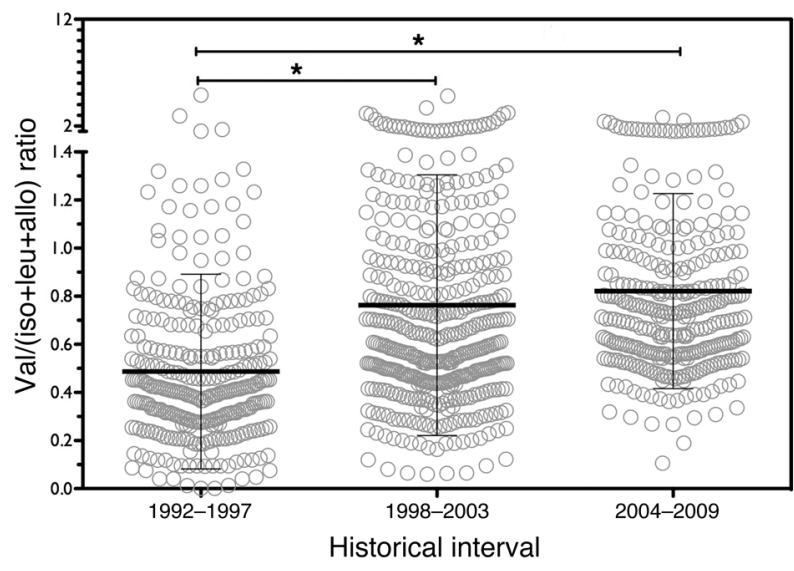

\section{Methods}

Study participants. We studied 26 MSUD patients managed with dietary therapy (MSUD diet; median age 19.5, range 7-35 years), 11 treated with liver transplantation (MSUD transplant; median age 12.0, range 5-26 years; surgery between 2.2 and 22.4 years of age, time since transplantation 0.2 to 14.0 years), and 26 age-matched controls (median age 15.9, range 6-35 years), most of whom were siblings to MSUD patients (Table 1). Twenty-six subjects were Old Order Mennonite and homozygous for the BCKDHA c.1312T>A mutation (39).

Neurochemical quantification. Neurochemical spectra were generated with single voxel proton MRS (echo time $=30 \mathrm{~ms}$; repetition time $=2000 \mathrm{~ms}$ ) using a point resolved spectroscopy pulse sequence on a Siemens Magnetom Trio 3 Tesla scanner over 80 acquisitions (40). Regions of interest were the left basal ganglia, midline prefrontal and anterior cingulate cortex, and right parieto-occipital white matter (Figure 8A), chosen based on their relevance to acute MSUD neuropathology $(12,41)$ and psychiatric illness $(34,36)$.

MRS spectra (Figure 8B) were quantified using LCModel (s-provencher. com/pages/lcmodel.shtml) with standard brain water referencing and

\section{Figure 8}

MRS methods. Regions of interest for quantitative MRS were placed in the left basal ganglia (A, left), prefrontal and anterior cingulate cortices (middle), and right parietal centrum semiovale white matter (right). Sample resulting MRS spectra from the anterior cingulated cortex of a control (B, left) and MSUD patient (B, right) demonstrate a relative decrease in glutamate (Glu), creatine (Cre), and NAA peaks. The choline peak (Cho) is also labeled in the spectra from the MSUD patient.

\section{Figure 7}

Historical improvement in cerebral valine nutrition. Improved dietary management has resulted in a trend of an increasing ratio of valine (val) to the other BCAAs (isoleucine, leucine, allo-isoleucine [allo]) competing for transport across the blood brain barrier. ${ }^{*} P<0.05$. Error bars represent mean $\pm S D$. correction for voxel cerebrospinal fluid composition $(42,43)$. Metabolites that varied more than $20 \%$ of the mean across 80 averages due to artifact were excluded. Using this criterion, we quantified NAA, creatine, choline compounds (primarily glycerophosphocholine and phosphocholine) (33), myo-inositol, and glutamate in millimoles per kilogram of brain tissue.

Neuropsychiatric testing. Full scale, verbal, and performance IQs were obtained using the Wechsler Abbreviated Scale of Intelligence (44). Determination of depression, anxiety, ADHD, and global function were guided by the Structured Clinical Interview for the Diagnostic and Statistical Manual of Mental Disorders (DSM-IV) (45) or its childhood version (46). Global assessment of function, a scale of social, occupational, and psychological functioning described in the DSM-IV, was also determined through this interview.

Age-appropriate continuous measures for depression and anxiety were drawn from the Beck Depression (47) and Anxiety (48) Inventories or sub-scores of the Beck Youth Inventories of Emotional and Social Impairment (49). Scaled scores for adult and youth versions were reconciled by calculating $\mathrm{z}$-scores, where $\mathrm{z}$ = (patient score - age-adjusted mean score)/
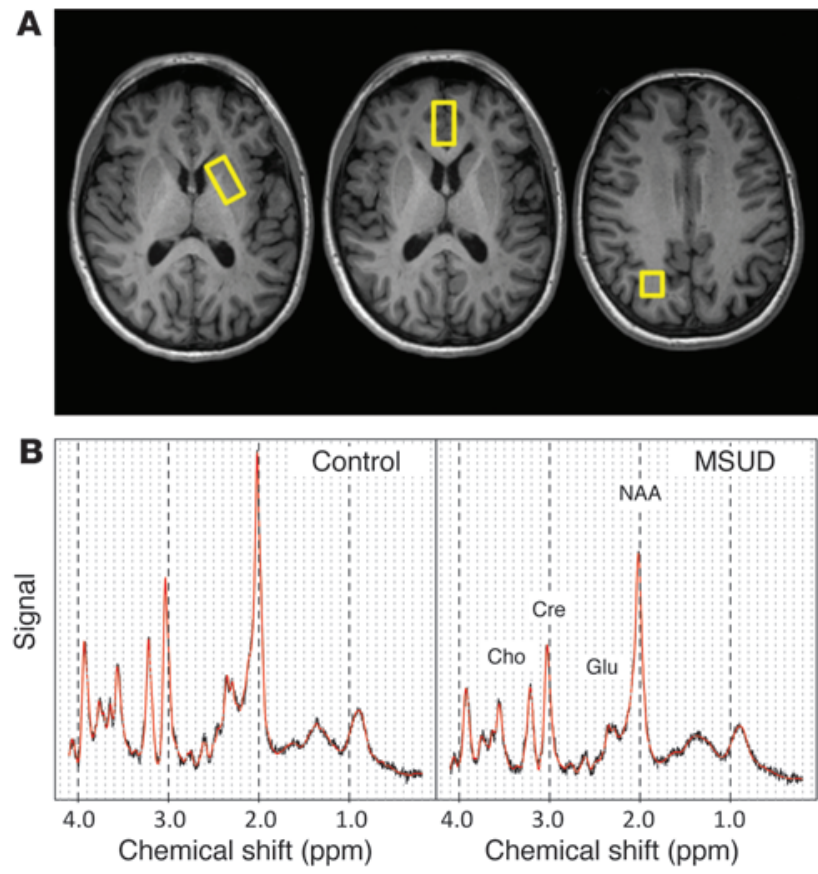
(age-adjusted SD) (50, 51). Conners' Parent Rating Scale-Revised (52), completed by spouses in the case of married participants, were used as a continuous measure of ADHD symptomatology using hyperactive-impulsive, inattentive, and total subscores based on DSM-IV criteria. For 5 participants ( 2 controls, 3 MSUD), no family member was available to complete the ADHD questionnaire.

Metabolic control and cerebral influx modeling. Samples for plasma amino acid quantification were obtained from $92 \%$ of participants the day of neuroimaging (15), Cerebral leucine influx was estimated using published Michaelis-Menten constants for the cerebral endothelial large amino acid transporter-1 (LAT1) (2). For 27 subjects (22 diet, 5 transplant), leucine levels were obtained within 2 weeks of neuropsychiatric evaluation.

Sufficient data were available to determine early life course and subsequent longitudinal control in a subgroup of 23 Mennonite MSUD patients. To characterize longitudinal essential amino acid homeostasis, we used frequency of amino acid monitoring (levels/yr), BCAA levels, and leucine variability measured across the life span (with a minimum gap of 5 days between consecutive samples to avoid oversampling during illness), and mean lifetime plasma ratios of leucine, the strongest BCAA competitor at the blood-brain barrier $\left(K_{m}\right.$ for LAT1 $\left.=29 \mu \mathrm{M}\right)$, to other amino acids of interest, specifically valine $\left(K_{m}=210 \mu \mathrm{M}\right)$, tyrosine $\left(K_{m}=64 \mu \mathrm{M}\right)$, tryptophan $\left(K_{m}=15 \mu \mathrm{M}\right)$, and methionine $\left(K_{m}=40 \mu \mathrm{M}\right)$.

Statistics. Fisher's exact test was used for categorical comparisons. Neuropsychiatric measures were compared across groups using 3-way ANOVA. Neurometabolites were compared using a 3-way, type-III analy- sis of covariance with age and partial volume as covariates. Post hoc Tukey's tests identified group differences. A $P$ value of less than 0.05 was considered significant. Neurometabolites with a nonnormal distribution using the Shapiro-Wilk test were transformed using a $\log _{10}$ scale. Spearman nonparametric correlations $\left(r_{s}\right)$ were used to relate neuropsychiatric outcomes and metabolic control. To control for age and partial volume effects, a partial correlation coefficient was calculated for correlations involving neurochemistry.

Study approval. The study was approved by the Penn State College of Medicine Institutional Review Board. Participants (or their parents) consented in writing to participation in the study and publication of the research.

\section{Acknowledgments}

We would like to thank Megan Taylor Sutton, Erik G. Puffenberger, and Pavlina Todorova for their assistance in data collection. This work was supported by the Pennsylvania State University Department of Psychiatry and the Penn State Hershey Neurosciences Institute.

Received for publication October 22, 2012, and accepted in revised form January 17, 2013.

Address correspondence to: Emilie Muelly, P.O. Box 850, MC \#161, Hershey, Pennsylvania 17033, USA. Phone: 412.983.0410; Fax: 815.301.8942; E-mail: emilie@muelly.com.
1. Strauss KA, Puffenberger EG, Morton DH. Maple syrup urine disease. In: Pagon R, Bird T, Dolan C, Stephens, K, Adam, M eds. GeneReviews. Seattle, Washington, USA: University of Washington; 2006.

2. Strauss KA, et al. Classical maple syrup urine disease and brain development: Principles of management and formula design. Mol Genet Metab. 2010; 99(4):333-345.

3. Strauss KA, et al. Elective liver transplantation for the treatment of classical maple syrup urine disease. Am J Transplant. 2006;6(3):557-564.

4. Shellmer DA, et al. Cognitive and adaptive functioning after liver transplantation for maple syrup urine disease: a case series. Pediatr Transplant. 2011; 15(1):58-64.

5. Mazariegos GV, et al. Liver transplantation for classical maple syrup urine disease: long-term followup in 37 patients and comparative United Network for Organ Sharing experience. J Pediatr. 2012; 160(1):116-121.e1.

6. Hutson SM, et al. Role of branched-chain aminotransferase isoenzymes and gabapentin in neurotransmitter metabolism. J Neurochem. 1998;71(2):863-874.

7. Hutson SM, Lieth E, LaNoue KF. Function of leucine in excitatory neurotransmitter metabolism in the central nervous system. J Nutr. 2001;131(3):846S-850S.

8. Dodd PR, et al. Glutamate and gamma-aminobutyric acid neurotransmitter systems in the acute phase of maple syrup urine disease and citrullinemia encephalopathies in newborn calves. J Neurochem. 1992; 59(2):582-590.

9. Zinnanti WJ, et al. Dual mechanism of brain injury and novel treatment strategy in maple syrup urine disease. Brain. 2009;132(pt 4):903-918.

10. Prensky AL, Moser HW. Brain lipids, proteolipids, and free amino acids in maple syrup urine disease. J Neurochem. 1966;13(9):863-874.

11. Felber SR, Sperl W, Chemelli A, Murr C, Wendel U. Maple syrup urine disease: metabolic decompensation monitored by proton magnetic resonance imaging and spectroscopy. Ann Neurol. 1993; 33(4):396-401.

12. Jan W, et al. MR diffusion imaging and MR spectroscopy of maple syrup urine disease during acute metabolic decompensation. Neuroradiology. 2003; 45(6):393-399.

13. Sener RN. Maple syrup urine disease: diffusion $\mathrm{MRI}$, and proton MR spectroscopy findings. Comput Med Imaging Graph. 2007;31(2):106-110.

14. Righini A, Ramenghi LA, Parini R, Triulzi F, Mosca F. Water apparent diffusion coefficient and T2 changes in the acute stage of maple syrup urine disease: evidence of intramyelinic and vasogenic-interstitial edema. J Neuroimaging. 2003;13(2):162-165.

15. Morton DH, Strauss KA, Robinson DL, Puffenberger EG, Kelley RI. Diagnosis and treatment of maple syrup disease: a study of 36 patients. Pediatrics. 2002;109(6):999-1008.

16. Berry GT, et al. Branched-chain amino acid-free parenteral nutrition in the treatment of acute metabolic decompensation in patients with maple syrup urine disease. N Engl J Med. 1991;324(3):175-179.

17. Kaplan P, et al. Intellectual outcome in children with maple syrup urine disease. J Pediatr. 1991; 119(1 pt 1):46-50.

18. Zielke HR, Zielke CL, Baab PJ, Collins RM. Large neutral amino acids auto exchange when infused by microdialysis into the rat brain: implication for maple syrup urine disease and phenylketonuria. Neurochem Int. 2002;40(4):347-354.

19. Fernstrom JD. Large neutral amino acids: dietary effects on brain neurochemistry and function [published online ahead of print June 8, 2012]. Amino Acids. doi:10.1007/s00726.012.1330-y.

20. Zielke HR, et al. Effect of alpha-ketoisocaproate and leucine on the in vivo oxidation of glutamate and glutamine in the rat brain. Neurochem Res. 1997; 22(9):1159-1164.

21. Yudkoff $\mathrm{M}$, et al. Interrelationships of leucine and glutamate metabolism in cultured astrocytes. $\mathrm{J} \mathrm{Neu}$ rochem. 1994;62(3):1192-1202.

22. Yudkoff M. Brain metabolism of branched-chain amino acids. Glia. 1997;21(1):92-98.

23. Clark JB, Land JM. Differential effects of 2-oxo acids on pyruvate utilization and fatty acid synthesis in rat brain. Biochem J. 1974;140(1):25-29.

24. Dreyfus PM, Prensky AL. Further observations on the biochemical lesion in maple syrup urine dis- ease. Nature. 1967;214(5085):276.

25. Jackson RH, Singer TP. Inactivation of the 2-ketoglutarate and pyruvate dehydrogenase complexes of beef heart by branched chain keto acids. J Biol Chem. 1983;258(3):1857-1865.

26. Wajner A, et al. Synaptic plasma membrane $\mathrm{Na}(+)$, $\mathrm{K}(+)$-ATPase activity is significantly reduced by the alpha-keto acids accumulating in maple syrup urine disease in rat cerebral cortex. Metab Brain Dis. 2007; 22(1):77-88.

27. Yudkoff $\mathrm{M}$, et al. Astrocyte leucine metabolism: significance of branched-chain amino acid transamination. J Neurochem. 1996;66(1):378-385.

28. Kamei A, Takashima S, Chan F, Becker LE. Abnormal dendritic development in maple syrup urine disease. Pediatr Neurol. 1992;8(2):145-147.

29. Schönberger S, Schweiger B, Schwahn B, Schwarz $\mathrm{M}$, Wendel U. Dysmyelination in the brain of adolescents and young adults with maple syrup urine disease. Mol Genet Metab. 2004;82(1):69-75.

30. Möller HE, Weglage J, Wiedermann D, Ullrich K. Blood-brain barrier phenylalanine transport and individual vulnerability in phenylketonuria. J Cereb Blood Flow Metab. 1998;18(11):1184-1191.

31. Koch R, Moats R, Guttler F, Guldberg P, Nelson M. Blood-brain phenylalanine relationships in persons with phenylketonuria. Pediatrics. 2000; 106(5):1093-1096.

32. Gropman AL, et al. 1H MRS allows brain phenotype differentiation in sisters with late onset ornithine transcarbamylase deficiency (OTCD) and discordant clinical presentations. Mol Genet Metab. 2008;94(1):52-60.

33. Govindaraju V, Young K, Maudsley AA. Proton NMR chemical shifts and coupling constants for brain metabolites. NMR Biomed. 2000;13(3):129-153.

34. McRae K, et al. The neural bases of distraction and reappraisal. J Cogn Neurosci. 2010;22(2):248-262.

35. Cohen J. A power primer. Psychol Bull. 1992; 112(1):155-159.

36. Paloyelis Y, Mehta MA, Kuntsi J, Asherson P. Functional MRI in ADHD: a systematic literature review. Expert Rev Neurother. 2007;7(10):1337-1356.

37. Yang P, Wu M-T, Dung S-S, Ko C-W. Short-TE pro- 
ton magnetic resonance spectroscopy investigation in adolescents with attention-deficit hyperactivity disorder. Psychiatry Res. 2010;181(3):199-203.

38. Perlov E, et al. Reduced cingulate glutamate/glutamine-to-creatine ratios in adult patients with attention deficit/hyperactivity disorder - a magnet resonance spectroscopy study. J Psychiatr Res. 2007; 41(11):934-941.

39. Fisher CR, et al. Maple syrup urine disease in Mennonites. Evidence that the Y393N mutation in E1 alpha impedes assembly of the $\mathrm{E} 1$ component of branchedchain alpha-keto acid dehydrogenase complex. JClin Invest. 1991;88(3):1034-1037.

40. Bottomley PA. Spatial localization in NMR spectroscopy in vivo. Ann N Y Acad Sci. 1987;508:333-348.

41. Cavalleri F, Berardi A, Burlina AB, Ferrari F, Mavilla L. Diffusion-weighted MRI of maple syrup urine disease encephalopathy. Neuroradiology. 2002;44(6):499-502.

42. Provencher SW. Estimation of metabolite concen- trations from localized in vivo proton NMR spectra. Magn Reson Med. 1993;30(6):672-679.

43. Weber-Fahr W, et al. A fully automated method for tissue segmentation and CSF-correction of proton MRSI metabolites corroborates abnormal hippocampal NAA in schizophrenia. Neuroimage. 2002;16(1):49-60.

44. Clements GR. An abbreviated form of the Wechsler Intelligence Scale for Children. J Consult Psychol. $1965 ; 29: 92$

45. First MB, Spitzer RL, Gibbon M, Williams JBW. Structured clinical interview for DSM-IV-TR Axis I Disorders, Research Version, Non-Patient Edition. (SCID-I/NP). New York, New York, USA: Biometrics Research, New York State Psychiatric Institute; 2002.

46. Matzner F, Silva R, Silvan M, Chowdhury M, Nastasi L. Preliminary Test-retest Reliability of the KID-SCID. Scientific Proceedings, American Psychiatric Association Meeting. Chicago, Illinois, USA. 1997.
47. Beck AT, Ward CH, Mendelson M, Mock J, Erbaugh J. An inventory for measuring depression. Arch Gen Psychiatry. 1961;4:561-571.

48. Beck A, Steer R. Manual for the Beck Anxiety Inventory. San Antonio, Texas, USA: The Psychological Coorporation Harchourt Brace \& Company; 1993.

49. Beck J, Beck A, Jolly J. Beck Youth Inventories of Emotional and Social Impairment. San Antonio, Texas, USA: The Psychological Coorporation; 2001.

50. Gillis MM, Haaga DAF, Ford GT. Normative values for the Beck Anxiety Inventory, Fear Questionnaire, Penn State Worry Questionnaire, and social phobia and anxiety inventory. Psychol Assess. 1995;7(4):450-455.

51. Seggar LB, Lambert MJ, Hansen NB. Assessing clinical significance: Application to the beck depression inventory. Behavior Therapy. 2002;33(2):253-269.

52. Conners CK. Rating scales in attention-deficit/hyperactivity disorder: use in assessment and treatment monitoring. JClin Psychiatry. 1998;59(suppl 7):24-30. 